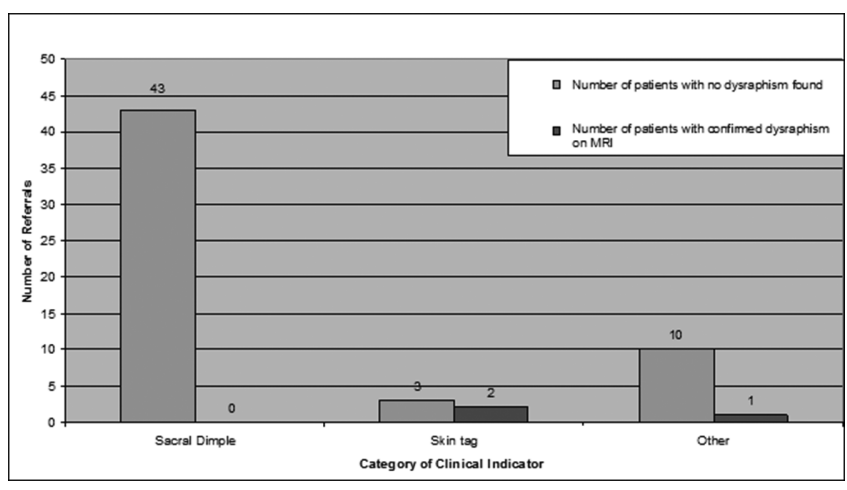

Abstract G298(P) Figure 2 Total referrals for clinical indicators showing patients with Dysraphism on MRI

Total Referrals for Clinical Indicators Showing Patients with Dysraphism on MRI - Figure 2

Conclusions The presence of a simple sacral dimple is a poor marker for spinal dysraphism, and as a result we present a new guideline for use. By reducing over-investigation of babies with sacral dimples, there is scope for potential saving on time, resources, inconvenience to parents and cost.

\section{G299(P) AWARENESS LEVELS OF THE PUBLIC IN DERBYSHIRE ABOUT THE QUALITY OF MEDICINES}

Tariq Almuzaini, Imti Choonara, Helen Sammons. Academic Division of Child Health, University of Nottingham, Derby, UK

\subsection{6/archdischild-2015-308599.276}

Aims The rationale for this study was to explore public' 1) willingness to consider the possibility of defective medicines; 2) awareness of the Yellow Card Scheme (YCS) to report any safety issues regarding medicines; and 3) views on purchasing medicines from online pharmacies and their awareness about the official logo of registered online pharmacies in the UK as the only mean to identify legitimate online pharmacies in the UK.

Methods This was a questionnaire study containing case scenarios derived from actual reported incidents of defective medicines from the MHRA. The questionnaire was piloted with 14 members of the public. All adults attending the outpatient department of the Derbyshire Children's Hospital, with or without a child, were considered as potential participants.

Results 400 questionnaires were completed and returned (response rate 90\%). Only a few participants (8\%) considered the possibility of manufacturer error in the first scenario when the defect was obscure (ibuprofen containing antipsychotic drugs due to packaging errors). The percentage increased to $37 \%$ in the second scenario when the defect was more obvious (an antihistamine with musty and mouldy odour). In both cases, most participants preferred to report complaints to healthcare professionals. Only $4 \%$ of the participants were aware of the YCS. More than one-third of the respondents (35\%) felt that online pharmacies are convenient in terms of buying medicines and $41 \%$ of them said that they will consider buying medications from online pharmacies if they were sold at cheaper prices. However, only 9 (2\%) respondents were aware of the official logo for legitimate and registered online pharmacies.

Conclusions The survey results showed that members of the public, in Derbyshire, were not aware about the possibility of defective medicines or that they can report via the YCS. Furthermore, despite the growing acceptance of using online pharmacies to obtain medicines, participants were unable to identify legitimate pharmacies and therefore are vulnerable to the risk of purchasing defective medicines. This study recommends more public campaigns to increase awareness of YCS and the official online pharmacy logo.

\section{G300(P) EMPIRICAL ACICLOVIR IN THE MANAGEMENT OF FEBRILE NEONATES}

${ }^{1} \mathrm{MA}$ Iro, ${ }^{2,3,4,8} \mathrm{M}$ Tebruegge, ${ }^{5,6} \mathrm{E}$ Pelosi, ${ }^{2,3,4,7} \mathrm{~S}$ Faust, ${ }^{3} \mathrm{~S}$ Patel. ${ }^{1}$ Oxford Vaccine Group, Department of Paediatrics, University of Oxford and the National Institute for Health Research Oxford Biomedical Research Centre, Oxford, UK; ${ }^{2}$ Academic Unit of Clinical and Experimental Sciences, Faculty of Medicine, University of Southampton, Southampton, UK; ${ }^{3}$ Department of Paediatric Immunology and Infectious Diseases, Southampton Children's Hospital, University Hospital Southampton NHS Foundation Trust, Southampton, UK; ${ }^{4}$ National Institute for Health Research Wellcome Trust Clinical Research Facility, University Hospital Southampton NHS Foundation Trust, Southampton, UK; ${ }^{5}$ Department of Infection, University Hospital Southampton NHS Foundation Trust, Southampton, UK; ${ }^{6}$ Public Health England Microbiology Services, Southeast Regional Laboratory, Southampton, UK; ${ }^{7}$ Institute for Life Sciences, University of Southampton, Southampton, UK; ${ }^{8}$ Department of Paediatrics, The University of Melbourne, Parkville, Australia

\subsection{6/archdischild-2015-308599.277}

Background Neonatal herpes simplex infection can result in serious morbidity and mortality. This study assessed the attitudes of paediatric doctors towards the use of empirical aciclovir in the management of febrile neonates.

Methods Invitations to participate in an online survey were sent to paediatric trainees (at senior house officer (SHO) and specialist registrar $(\mathrm{SpR})$ level) and consultants $(\mathrm{n}=513)$ in two medical deaneries in the UK (Wessex and Oxford Deanery) between May and June 2013. Four hypothetical scenarios of a febrile neonate were described to represent disseminated HSV disease (scenario 1), low-risk for HSV (scenario 2), HSV skin, eye and mouth disease (scenario 3) and HSV central nervous system disease (scenario 4). Participants were asked to indicate which antimicrobials they would commence for each scenario.

Findings A total of 64 individuals participated (response rate: $12.5 \%)$. A substantial proportion of participants indicated they would not commence aciclovir despite the presence of clinical and/or laboratory features suggestive of neonatal HSV infection in scenarios $1(34 / 63,54 \%), 3(24 / 57,42 \%)$ and $4(31 / 54,57 \%)$. Conversely, a total of $7 / 60$ participants (12\%) indicated they would start aciclovir in the scenario with low probability of HSV disease (scenario 2). Throughout, there was a trend for a higher proportion of consultants choosing to commence aciclovir compared with trainees; however, this was not statistically significant.

Conclusion The results indicate that there is lack of awareness of key features suggestive of neonatal herpes simplex infection, and highlight the need to integrate guidance on the use of empirical aciclovir in febrile neonates into national guidelines.

\section{G301(P) MEDICAL INTERVENTION FOR CHILDREN WITH MEDICAL COMPLEXITY (MICMAC)}

ZS Al-Harthy, JP Cowling, GK Mann, M Salama. Birmingham Children's Hospital, Birmingham, UK

10.1136/archdischild-2015-308599.278 
Aim To evaluate the effect of targeted consultant time in sharing mental models with parents and nursing staff for children with medical complexity (CMC).

Background CMC are part of a mixed caseload covered by the acute paediatric team. We define them here as children with a length of stay $\geq 7$ days with $\geq 2$ system involvement. Feedback shows these children experience prolonged length of stays (LOS) with a need for coordination of care often between different teams. Parents have highlighted that that better communication with them, within teams and between different teams could improve their child's journey.

Methods All team members were briefed using written and verbal communication as were parents and children taking part. Patients were allocated a weekly slot over a four week period to meet with the MICMAC consultant and a member of the nursing team. Sharing of mental models was facilitated using a targeted proforma covering current expectations and barriers to discharge. The MICMAC consultant was briefed by the service team prior to the meeting and handed back afterwards. Qualitative feedback was obtained using structured debriefing interviews with ward managers and parents. Group debriefing sessions were carried out with the junior medical team and the consultant body covering key themes highlighted by the parental feedback.

Results A total of 12 patients with an average LOS of 90 days (range 13-241) over 6 wards were included. 11 consultants led a total of 34 meetings. 3 children were discharged home and 2 patients had withdrawal of care. A total of 6 nursing manager interviews and 9 parental interviews and one substituted lead clinician interview were undertaken. Debriefing of the medical teams was undertaken in 2 group sessions. Positive feedback was centred around improved coordination of care, understanding of overall direction and sharing of information.

Conclusions The CMC group is recognised as an expanding patient group. Key factors influencing their inpatient journey involve communication between caregivers and the responsible teams. We highlight how a simple targeted communication tool can improve quality of patient journey.

\section{G302(P) OBESITY - WHY DO CLINICIANS STILL TURN A "BLIND EYE"}

L Ek, T Waterfield, R Shahid, C Lander, N Nathwani. Paediatrics, Luton and Dunstable Hospital, Luton, UK

\subsection{6/archdischild-2015-308599.279}

Approximately 3 in 10 children aged 2-15 years are overweight or obese. This is concerning because weight problems in childhood often continue into adulthood. NICE guidance states that healthcare professionals should 'aim to create a supportive environment that helps a child who is overweight or who has obesity, and their family, make lifestyle changes'.

Aims

1. Determine the prevalence of obesity amongst paediatric outpatients

2. Audit practice against NICE guidance

3. Collect qualitative data on clinician attitudes towards obesity

Methods This prospective audit assessed the weight, BMI, ethnicity and outcome of patients presenting to the paediatric outpatients department (POD) over a 10 week period. Patients were audited from all clinical specialities using the POD.

Overweight and obese children were identified as having a weight above the $91^{\text {st }}$ and $98^{\text {th }}$ centiles respectively and underweight children a BMI below the $0.4^{\text {th }}$ centile on the appropriate RCPCH Body Mass Index (BMI) charts and growth charts. The only exclusion criteria were children under 2 and over 16 years of age. Qualitative data was collected via structured interviews with clinicians working in the POD.

Results 569 children were audited of which 407 met the inclusion criteria. The cohort's demographics were as expected for the area. The prevalence of overweight and obese children from this cohort was $14 \%$ and $11 \%$ respectively. Of those identified as either overweight or obese only $2 \%$ and $34 \%$ respectively were offered an intervention. Interventions included; advice, investigations, or referral. Interestingly all underweight children were investigated and given follow up support. Qualitative data from structured interviews demonstrated that clinicians were aware of obesity as a health issue but failed to act for a variety of reasons including a lack of time and for fear of damaging the doctor-patient relationship.

Analysis Clinicians are not complying with NICE guidelines for a range of reasons including; time constraints, a lack of training and concerns over damaging the doctor-patient relationship. We are currently training an obesity nurse specialist to support clinicians during outpatient clinics and to offer additional training and educational support with a view to changing clinician attitudes towards obesity.

\section{G303(P) AWARENESS OF HEALTHCARE PROFESSIONALS ABOUT THE POSSIBILITY OF SUBSTANDARD MEDICINES}

T Almuzaini, I Choonara, H Sammons. Academic Division of Child Health, University of Nottingham, Derbyshire Children's Hospital, Derby, UK

\subsection{6/archdischild-2015-308599.280}

Aims The number of reports per year by the MHRA of substandard medicines is rising. The aim of this study was to examine healthcare professionals' (HCPs) willingness to consider and report defective medicines. To gauge their awareness of reporting systems designed for HCPs to report defective medicines, issues related to medicine quality and the official online pharmacy logo (as the marker of legitimate online pharmacies).

Methods This pilot study involved HCPs (paediatric doctors, pharmacists, and children's nurses) and used self-administered questionnaires containing case scenarios derived from actual reported incidents. An invitation letter and questionnaire were sent to each doctor and nurse working at Derbyshire Children's Hospital, as well as pharmacist members of the Nottinghamshire and Derbyshire Local Pharmacy Forum.

Results 30 doctors (60\% response rate), 31 pharmacists $(2.7 \%)$, and 47 nurses (42\%) responded, a total of 108 HCPs. Only 27 HCPs (23\% doctors, 39\% pharmacists, and 17\% nurses) considered the possibility of manufacturer error when a medicine's defect was obscure (fentanyl transdermal system with manufacturer error causing excessive release of the medicine). Most HCPs $(77 \%, 74 \%$, and 66\%) responded that they would report such an incident via the Yellow Card Scheme, established to gather reports of adverse drug reactions. Most HCPs (100\%, $87 \%$, and $81 \%$ ) agreed with the statement that 'medicines in the UK are manufactured to a high standard', though more than one-third (40\%, 39\%, and 30\%) believed that poor-quality medicines existed in the medicine supply chain in the UK. However, only $5 \%$ of HCPs were aware of the defective medicines reporting system and few $(7 \%, 16 \%$, and $6 \%)$ were aware of the official online pharmacy logo. 